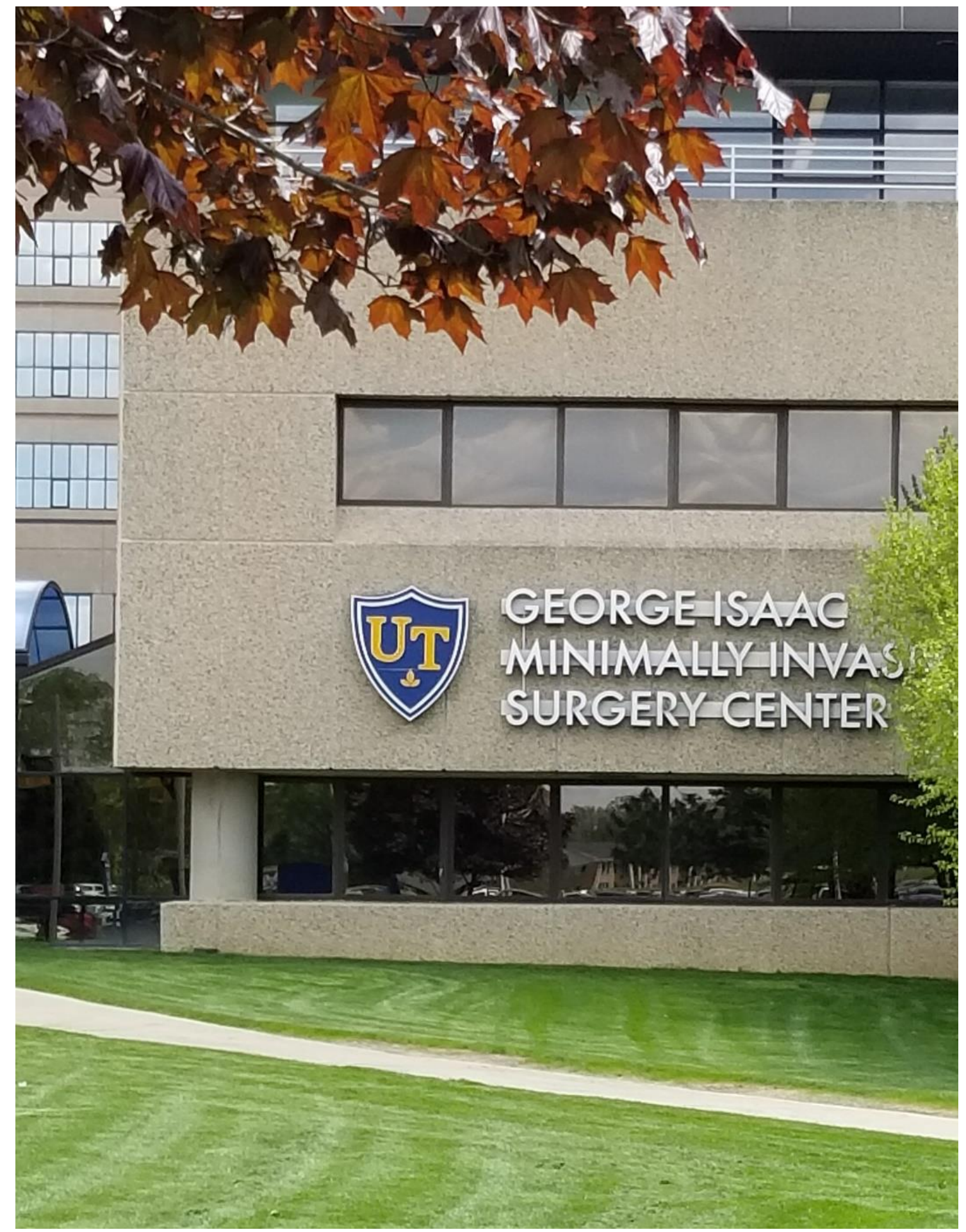




\title{
Critical hypomagnesemia and seizure induced by chronic use of proton pump inhibitors (PPIs)
}

\author{
Gheith Yousif, ${ }^{a, 1}$ Mohanad Baldawi, $^{a}$ Sarah Faisal ${ }^{a}$ \\ ${ }^{a}$ Department of Family Medicine, Health Science Campus, The University of Toledo, 3000 Arlington, Toledo OH 43614-2598, USA
}

\begin{abstract}
Background: Chronic use of proton pump inhibitors (PPIs) may lead to severe hypomagnesemia, although it is a rare side effect. Hypomagnesemia related to PPIs use less reported compared to other side effects. Critically low magnesium level may lead to fatal seizure activity, which could lead to death if went undiagnosed. Case presentation: This is a report of a 49-year old female with a history of gastroesophageal reflux disease (GERD) presented to our emergency department with a seizure activity and critically low magnesium level $(<0.5 \mathrm{mg} / \mathrm{dL}$ ). Initially the patient was hemodynamically unstable, required intubation, and admitted to the medical intensive care unit (MICU). After further management in MICU, patient stabilized and transferred to the inpatient regular medical floor. Most of the potential common causes of her low magnesium level were thoroughly investigated and ruled out except for PPIs use (as she was a chronic user). The patient was advised to discontinue her PPIs and to use alternative medications because of life-threatening side effect "hypomagnesemia" and based on the risk-benefit balance (as the risk overweight the benefits in this situation). No further hypomagnesemic episodes reported after the second admission to the ICU unit when PPIs were discontinued completely. Conclusion: Although PPIs use is beneficial for patients with GERD especially those with gastritis, but may lead to life-threatening hypomagnesemia in rare occasions. Physicians should be aware of this side effect in all patients with chronic PPIs use. In addition, we recommend that patients who developed this rare side effect need to use alternative medications to prevent recurrence and fatal consequences. Further research is needed to determine the incidence and the association between the development of hypomagnesemia and the use of different types of gastric acid suppressants.
\end{abstract}

PPIs | hypomagnesemia | seizure |

P roton Pump Inhibitors (PPIs) are commonly prescribed medications for the purpose of gastric acid related disorders like peptic ulcer disease, GERD, Helicobacter pylori infection, Zollinger-Ellison syndrome, Barret's esophagitis, and functional dyspepsia (1-5), also considered one of the most widely prescribed drugs in the world (1). The mechanism of action of PPIs is gastric acid secretion reduction by inhibition of $\mathrm{H}^{+}, \mathrm{K}^{+}$-ATPase proton pumps in the gastric parietal cells (6). Omeprazole, pantoprazole, lansoprazole, dexlansoprazole, rabeprazole, and esomeprazole (stereo isomer of omeprazole) are among the most common PPIs approved for use in the United States (6). Because of the immense success from treatment of gastric acid disorders by the use of PPIs, non-steroidal anti-inflammatory drug (NSAID) associated gastropathy incidence and indications for elective surgery for treatment of peptic ulcer disease have both been substantially reduced (5). Although these medications are generally considered safe, they are associated with development of significant side effects including Clostridium difficile-associated diarrhea, interstitial nephritis (very uncommon side effect), pneumonia, vitamin B12 deficiency, osteoporosis, and other musculoskeletal complications (e.g., hip fracture)
(1). In this report, we will describe this critical PPI induced hypomagnesemia side effect with seizure activity related to our patient.

\section{Case report}

Patient information. Caucasian female, 49 year old, weight 210 pounds and 62 inches height.

Objective for case reporting. In our report, we present the case of hypomagnesemia, due to the chronic use of over the counter PPI for gastric acid reflux.

Case. A 49-year-old female presented to University of Toledo Medical Center (UTMC) Emergency Department with altered mental status (initially was only arousable on verbal and painful stimuli), found to have critically low magnesium level $(<0.5 \mathrm{mg} / \mathrm{dL})$. Patient mentation deteriorated rapidly and developed seizure activity with acute hypoxic respiratory failure that required instant intubation. At that time potassium level was $3.1 \mathrm{mEq} / \mathrm{L}$, sodium $138 \mathrm{mEq} / \mathrm{L}$, creatinnie $1.3 \mathrm{mg} / \mathrm{dL}$, and her electrocardiogram showed normal sinus rhythm. Patient transferred to the medical intensive care unit (MICU) directly after intubation and mechanical ventilation. Laboratory work been done including: autoimmune panel, Helicobacterpylori serology, Vitamin D level, parathyroid level, pro-calcitonin, sepsis work-up (blood culture, urine culture, chest x-ray, IV fluid resuscitation, and early antibiotics started), Lipase, amylase, HbA1c, calcium level and urine analysis including urine toxicology screen were all within normal range. The patient had no history of alcohol or drug abuse.

The patient recently underwent cholecystectomy, and she had frequent vomiting and diarrhea. At the beginning, we thought her hypomagnesemia related simply to gastrointestinal loss "frequent diarrhea and vomiting", which may lead to contraction alkalosis and critically low magnesium levels. The patient's diarrhea and vomiting were resolved, and she was stabilized clinically and transferred to the inpatient medical floor to continue management per primary team. The patient improved and was discharged with advice to follow up with her primary care physician.

Despite resolution of her vomiting and diarrhea, she continued to have low magnesium levels. In fact, she required another admission to the same Emergency Department because of critically low magnesium level and altered mental status "same symptoms". She

All authors contributed to this paper. ${ }^{1}$ To whom correspondence should be sent Gheith.Yousif@UToledo.edu

The authors declare no conflict of interest. Submitted: May/7/2019, published: June/10//2019. Freely available online through the UTJMS open access option 
was re-admitted to the MICU again, but this time she did not require intubation, after correcting her magnesium level, her condition stabilized then transferred to the inpatient regular medical floor for further observation. Her condition improved then discharged home to follow up with her primary care physician. There was three months between the two admissions and both magnesium levels were $<0.5$ $\mathrm{mg} / \mathrm{dL}$. During the outpatient clinic visits, the causes of hypomagnesemia were thoroughly investigated to find out what was the major factor contributed to her condition as follows:

Gastrointestinal loss: diarrhea, malabsorption and steatorrhea, and small bowel bypass surgery.

Acute pancreatitis.

Medications: Mainly PPIs, diuretics, certain antibiotics (aminoglycoside, amphotericin, pentamidine), Calcineurin inhibitors, Cisplatin.

Genetic disorders: Intestinal hypomagnesemia with secondary hypocalcemia, Renal losses.

Antibodies targeting epidermal growth factor (EGF) receptor (e.g., cetuximab, panitumumab, matuzumab).

Volume expansion.

Uncontrolled diabetes mellitus.

Alcoholism.

Hypercalcemia.

Acquired tubular dysfunction.

Recovery from acute tubular necrosis.

Post-obstructive diuresis.

Post-renal transplantation.

Genetic disorders: Barter/Gitelman syndrome. cinosis

Familial hypomagnesemia with hypercalciuria and nephrocal-

Autosomal dominant isolated hypomagnesemia (Na-K-ATPase gamma subunit, Kv1.1 and cyclin M2 mutations). tion).

Autosomal recessive isolated hypomagnesemia (EGF muta-

Renal malformations and early-onset diabetes mellitus (HNF1beta mutation).

Not all the above mentioned were investigated because of the cost. From the long list only the genetic disorders chronic PPI use (Omeprazole) was on the top of our list. Gastrointestinal loss was also excluded because the patient continued to have low magnesium level during her second hospital admission despite the fact that she had no GI loss. In further workup, we found that the patient was taking a large amount of Omeprazole over the counter for uncontrolled acid-reflux. Our inpatient and outpatient workup for critically low magnesium level was negative for most of the above mentioned except for PPI use. Patient was new to our practice but mentioned that she have been on PPIs for many years and sometimes taking extra doses of Omeprazole $20 \mathrm{mg}$ in addition to her $40 \mathrm{mg}$ daily. For that we suggested her hypomagnesemic episodes might be due to chronic use of PPIs.

The patient was given magnesium tablet supplements for few days and counseled to discontinue PPI use Omeprazole with frequent magnesium level check until normalize. She also advised to follow life style modifications to improve her acid reflux and to use

1. Zipursky J, et al. (2014) Proton pump inhibitors and hospitalization with hypomagnesemia: a population-based case-control study. PLoS Med 11(9):e1001736.

2. Gröber U, et al. (2013) Magnesium in Prevention and Therapy. Nutrients 7(9):8199226.

3. Danziger J, et al. (2013) Proton-pump inhibitor use is associated with low serum magnesium concentrations. Kidney Int 83(4):692-9.

4. Trifan A, et al. (2017) Proton pump inhibitors therapy and risk of Clostridium difficile infection: Systematic review and meta-analysis. World $J$ Gastroenterol 23(35): 6500-6515.

4 | utdc.utoledo.edu/Translation alternative medications like $\mathrm{H} 2$ receptor blockers (e.g., Zantac). We were following up with the patient every 2 weeks for one month with magnesium supplements and to discontinue PPIs but use $\mathrm{H} 2$ blockers instead for her severe GERD. After discontinuation of the medication, adherence to life style modifications, and switching to $\mathrm{H} 2-$ receptor blockers, the patient did not have any recurrent episodes, and her magnesium levels during the follow-up period were within the normal range even without any magnesium supplements.

\section{Discussion}

Recent research suggests that PPIs may induce hypomagnesemia, but this relationship is only relevant in cases of long-term use, although these studies warned about the short-term use of less than 90 days $(5,7)$. The first cases of PPI-induced hypomagnesemia were reported in 2006 (1). In March of 2011, US Food and Drug Administration (FDA) reported that health care professionals should follow-up serum magnesium level for patients who are expected to be on long-term PPI use (3). The normal range of magnesium level in blood is 1.7 to $2.2 \mathrm{mg} / \mathrm{dL}$, and this level may be change according to the laboratory preferences. Magnesium plays a critical role by performing many functions in the body including protein synthesis, enzymatic reactions, and the regulation of ion channels (3). As a major component in many cellular reactions, there are numerous enzymes that are dependent on magnesium such as $\mathrm{Na}^{+} / \mathrm{K}^{+}$-ATPase, hexokinase, creatinine kinase and protein $\mathrm{ki}-$ nase (2). The homeostasis is regulated by intestinal absorption and renal excretion (3). PPIs cause hypomagnesemia due to intestinal malabsorption, as they have a potential inhibiting effect on TRMP6 transporter, which is the major pathway of intestinal absorption of magnesium (8). Magnesium deficiency can cause a wide range of symptoms such as loss of appetite, nausea, vomiting, fatigue and weakness, and it can also cause some life threatening complications such as tetany, convulsion, bradycardia, hypotension, and death $(2$, 3 ). Hypomagnesemia is known to cause different types of cardiac arrhythmia including atrial and ventricular tachycardia, prolonged QT interval and torsade de pointes (2). Physicians should be aware of these risks, particularly in patients on long-term use of PPI, and those with hypokalemia or associated cardiac or neurological symptoms (1).

\section{Conclusion}

Although PPI induced hypomagnesemia is rare side effect, it should not be under-estimated. It must be kept in consideration especially in patients using loop diuretics or in those with hypokalemia induced by other medical conditions (e.g., increased mineralocorticoid activity, non-reabsorbable anions, loss of gastric secretions, polyuria, renal tubular acidosis, Amphotericin B use, salt wasting neuropathies, Liddle's syndrome, Bartter and Gitelman syndromes, and low-calorie diet). Only a few cases of this side effect have been reported, failure to monitor and recognize this complication might lead to serious outcomes including seizure and probably death. On the other hand, early diagnosis and management of PPI induced hypomagnesemia by health care providers is considered very important and life-saving measure.
5. Scarpignato C, et al. (2016) Effective and safe proton pump inhibitor therapy in acid-related diseases - A position paper addressing benefits and potential harms of acid suppression. BMC Med 14(1):179.

6. Rouby N, Lima JJ, Johnson JA. (2018) Proton pump inhibitors: from CYP2C19 pharmacogenetics to precision medicine. Expert Opin Drug Metab Toxicol(4):447-460.

7. Park $\mathrm{CH}$, et al. (2014) The association between the use of proton pump inhibitors and the risk of hypomagnesemia: a systematic review and meta-analysis. PLoS One 9(11):e112558.

8. William JH, Danziger J, Proton-pump inhibitor-induced hypomagnesemia: Current research and proposed mechanisms, World J Nephrol 5(2):152-157. 